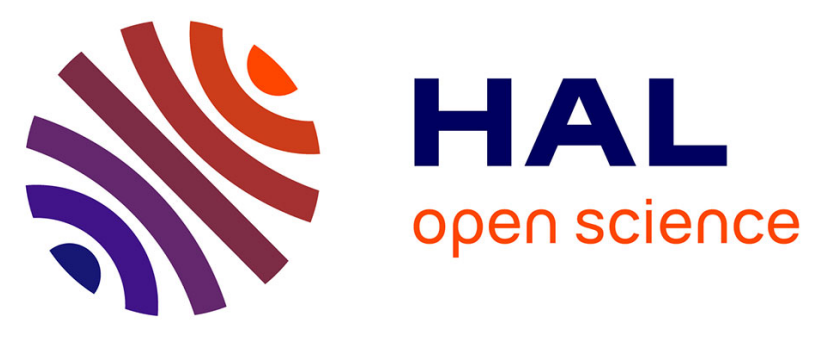

\title{
Abnormal respiratory cilia in non-syndromic Leber congenital amaurosis with CEP290 mutations
}

Jean Francois Papon, Isabelle Perrault, Andre Coste, Bruno Louis, Xavier

Gerard, Sylvain Hanein, Lucas Fares-Taie, Sylvie Gerber, Sabine

Defoort-Dhellemmes, Anne Marie Vojtek, et al.

\section{To cite this version:}

Jean Francois Papon, Isabelle Perrault, Andre Coste, Bruno Louis, Xavier Gerard, et al.. Abnormal respiratory cilia in non-syndromic Leber congenital amaurosis with CEP290 mutations. Journal of Medical Genetics, 2010, 47 (12), pp.829. 10.1136/jmg.2010.077883 . hal-00560778

\section{HAL Id: hal-00560778 https://hal.science/hal-00560778}

Submitted on 30 Jan 2011

HAL is a multi-disciplinary open access archive for the deposit and dissemination of scientific research documents, whether they are published or not. The documents may come from teaching and research institutions in France or abroad, or from public or private research centers.
L'archive ouverte pluridisciplinaire HAL, est destinée au dépôt et à la diffusion de documents scientifiques de niveau recherche, publiés ou non, émanant des établissements d'enseignement et de recherche français ou étrangers, des laboratoires publics ou privés. 


\section{Abnormal respiratory cilia in non-syndromic Leber congenital amaurosis with CEP290 mutations}

JF Papon ${ }^{1,2,3,4,5^{*}}$, I Perrault ${ }^{6^{*}}$, A Coste ${ }^{1,2,3,4}$, B Louis ${ }^{1}$, X Gérard ${ }^{7}, \mathrm{~S}$ Hanein ${ }^{6}, \mathrm{~L}$ Fares-Taie ${ }^{6}$, S Gerber ${ }^{6}$, S Defoort-Dhellemmes ${ }^{8}$, Anne Marie Vojtek ${ }^{9}$, J Kaplan ${ }^{6}$, JM Rozet ${ }^{6}$, Escudier $\mathrm{E}^{1,9,10,11}$

1. INSERM, Unit U955, Creteil, F-94010, France;

2. Universite Paris-Est Creteil Val de Marne, Faculte de Medecine, UMR_S955, Creteil, F-94010, France;

3. AP-HP, Groupe Henri-Mondor - Albert Chenevier, service d'ORL et de chirurgie cervico-faciale, Creteil, F-94010, France;

4. Hopital intercommunal, service d'ORL et de chirurgie cervico-faciale, Creteil, F-94010, France;

5. INSERM, Unit U933 Paris, F-75012, France;

6. Unité de Recherches en Génétique et Epigénétique des Maladies Métaboliques, Neurosensorielles et du Développement, INSERM U781 and Université Paris Descartes, CHU Necker Enfants Malades, Paris, France ;

7. Genethon Evry, France ;

8. Service d'Ophtalmogie, CHRU Roger Salengro, Lille, France ;

9. Hopital intercommunal, service d'anatomo-pathologie (laboratoire de microscopie electronique), Creteil, F-94010, France;

10. Universite Paris 6, Faculte de Medecine, Paris, F-75013, France;

11. AP-HP, Hopital Armand-Trousseau, service de genetique et d'embryologie medicales, Paris, F75012, France.

*JF Papon and I Perrault contributed equally to this work

Correspondance to Jean-Michel Rozet :

Postal address Hopital Necker-Enfants Malades

149 rue de Sèvres 


\section{Paris CEDEX 15}

E-mail : jean-michel.rozet@inserm.fr

Telephone number : + 33144495156

Fax number : + 33144495150

Key words : LCA, CEP290, ciliopathies, axonemal ultrastructure, ciliary movement Word count: $3632 / 4000$

Competing Interest: None declared. 


\section{ABSTRACT (241/250 words)}

Background : Leber congenital amaurosis (LCA) is the earliest and most severe inherited retinal degeneration. Isolated forms of LCA frequently result from mutation of the CEP290 gene which is expressed in various ciliated tissues.

\section{Methods :}

Seven LCA patients with CEP290 mutations were investigated to study otorhinolaryngologic phenotype and respiratory cilia. Nasal biopsies and brushing were performed to study cilia ultrastructure using transmission electron microscopy and ciliary beating using high-speed videomicroscopy, respectively. CEP290 expression in normal nasal epithelium was studied using real time RT-PCR.

\section{Results :}

When electron microscopy was feasible (5/7), high levels of respiratory cilia defects were detected. The main defects concerned dynein arms, central complex and/or peripheral microtubules. All patients had a rarefaction of ciliated cells and a variable proportion of short cilia. Frequent but moderate and heterogeneous clinical and ciliary beating abnormalities were found. CEP290 was highly expressed in the neural retina and nasal epithelial cells compared to other tissues.

\section{Discussion :}

These data provide the first clear demonstration of respiratory cilia ultrastructural defects in LCA patients with CEP290 mutations. The frequency of these findings in LCA patients along with the high expression of CEP290 in nasal epithelium suggest that CEP290 has an important role in the proper development of both the respiratory ciliary structures and the connecting cilia of photoreceptors. The presence of respiratory symptoms in patients could represent additional clinical criteria to direct CEP290 genotyping of patients affected with the genetically heterogeneous cone-rod dystrophy subtype of LCA. 


\section{INTRODUCTION}

Cilia are evolutionarily conserved microtubule-based structures found in most of the cells. They are involved in various biological processes such as motility, sensory perception and signalling.[1] Broadly speaking, there are two types of cilia, i.e. motile and primary cilia in which the core cytoskeleton, named axoneme, slightly differ. Typical motile cilium consists of a central pair of single microtubules surrounded by nine outer-doublet of microtubules. On each outer-doublet, inner and outer dynein arms and radial spokes are anchored (known as the "9+2" organization). The axoneme of primary non-motile cilia differs from motile cilia as there is no pair of central microtubule singlets and no dynein arms on the outer doublet microtubules. This is not true in the embryonic node where primary cilia are involved in the left-right asymmetry. Assembly, maintenance and function of motile and primary cilia involve evolutionary conserved bidirectional intraflagellar transport (IFT) of proteins complexes (motor proteins, IFT particules and their associated cargo) between the basal body and the distal tip of the axoneme.[2]

Ciliopathies refer to disorders accounted for by IFT alterations resulting from structural or functional alterations of the axoneme and/or basal bodies of primary and/or motile cilia.[3] Retinal ciliopathies are a group of hereditary retinal dystrophies affecting IFT in photoreceptors.[4] Vertebrate photoreceptors are polarized sensory neurons composed of an inner and an outer segment connected by a highly specialized primary cilium, the connecting cilium. The biogenesis and function of photoreceptors are highly dependent on IFT. Indeed, the synthesis of materials required for the formation and maintenance of outer segment occurs in the inner segment. Besides, adaptation to light relies on light-driven translocation of phototransduction regulatory proteins between the inner and outer segments.

IFT impairment in photoreceptors has recently proven its major importance in the pathophysiology of the earliest and most severe inherited retinal dystrophy, Leber 
congenital amaurosis (LCA) (MIM204000). As a matter of fact, 4 genes out of 13 so far identified LCA genes encode proteins located in photoreceptor connecting cilia or basal bodies: RPGRIP1,[5] TULP1,[6] CEP290,[7] and LCA5.[8] Conversely to RPGRIP1 and TULP1 which are specifically or preferentially expressed in the retina,[5, 6] LCA5 and CEP290 are expressed in various ciliated tissues.[7, 8] To our knowledge, LCA5 mutations were reported in few patients only affected with nonsyndromic LCA. [8, 9] Yet, CEP290 mutations were shown to be a frequent cause of non-syndromic LCA as well as syndromic LCA including Joubert syndrome, (JBTS5, MIM610188) Senior-Loken syndrome, (SLSN6, MIM610189) Meckel syndrome (MKS4, MIM611134) and possibly Bardet-Bield syndrome (BBS14, MIM610142). MKS4, JBTS5 and SLSN6 were consistently ascribed to loss-of-function CEP290 mutations.[10-12] Most patients affected with non-syndromic forms of LCA carry a intronic mutation of CEP 290 regarded by some authors as hypomorphic (c.2991+1655A>G;[7, 13]). However, some other patients were reported to harbour homozygous or compound heterozygous loss-of-function alleles.[13]

The pleiotropic phenotype accounted for by CEP290 mutations is consistent with the location of the protein in the primary cilia of photoreceptor cells, in the kidney and cerebellum. In the retina, CEP290 associates with several microtubule-based transport proteins including retinitis pimentosa GTPase-regulator (RPGR) which mutations are responsible for ca. $10 \%$ of X-linked retinitis pigmentosa (RP) (MIM312610). Interestingly, some syndromic RP phenotypes consistent with motile and primary cilia abnormalities were associated with RPGR mutations.[14-17] Here, we report that LCA patients with CEP290 mutations, including the intronic c. $2991+1655 A>G$ change, exhibit high levels of airway motile cilia defects and frequent respiratory symptoms. The frequency of these findings in LCA patients along with the high expression levels of CEP290 in the neural retina and nasal 
ciliated cells compared to other tissues suggest that the respiratory symptoms in LCA patients may not be coincidental.

\section{PATIENTS AND METHODS}

\section{LCA patients with CEP290 mutations}

Seven patients ( 2 adults, 5 children; age range 9-44 yrs; mean age $17.5 \mathrm{yrs;}$ Table 1) from six unrelated French families were ascertained from the Genetic and Ophthalmologic Departments of the University Hospitals of Paris Necker - Enfants Malades and Lille. All patients fulfilled the minimal criteria for the diagnosis of LCA described elsewhere.[18]

No patient had sign of known ciliopathy (visceral malposition, cystic formation in kidney, liver or pancreas...) but one patient presented with hypofertility due to asthenospermia (Patient 5, Table 1). The CEP290 genotype had been determined prior to the present study by direct sequencing of the $3^{\prime}$ and $5^{\prime}$ UTR, the coding region and exon-intron boundaries of the gene as described elsewhere (Table 1).[13] One out the patients was homozygous for the intronic CEP290 c.2991+1655A>G mutation while four out of them were compound heterozygous for this intronic change and a mutation presumably resulting in loss-of-function. Finally, two patients were homozygous for the p.Lys1575X mutation which may be regarded as a loss-of function allele according to i) the absence of splice change predicted by the Splice Site Prediction Program available at http://fruitfly.org/cgi-bin/seq tools/splice.pl and ii) the amplification of a unique transcript identical to the wild-type transcript (primers designed in exons 33 and 37, respectively; data not shown available on request).

\section{Rhino- and Otologic phenotype characterization}

The patients were examined by an otorhinolaryngologist who firstly recorded clinical history of airway diseases by interviewing the patients and/or their parents. Five different nasal symptoms (nasal obstruction, hypo-anosmia, rhinorrhea, nasal 
hyperreactivity (itching, pruritus) and facial pain) and three different otological symptoms (hearing loss, otorrhea and otalgia) were assessed according to a fourpoint semiquantitative scale: 0 , no symptom; 1 , moderate; 2 , mild symptom that slightly interfere with daily activities or sleep; 3 , severe symptom that severely interfere with daily activities or sleep. For each patient, a global symptom score was determined by summing the scores of each symptom, with a maximum rhinologic and otologic symptom scores of 15 and of 9 , respectively.[19]

Olfactory function of each patient was evaluated with the Brief Smell Identification test (B-SIT) as already reported.[20] The subjects were presented with a freshly scratched odor-impregnated panel a maximum of twice before being required to make a forced choice from 4 possible alternative answers. The numbers of correct answers from 12 possible correct answers were summed and scores $>8$ were considered normal and scores $\leq 8$ were considered abnormal.[21]

Audiometric evaluation included the air-conduction thresholds, bone-conduction thresholds and the air-bone gap (difference between air- and bone-conduction thresholds), when present. We used a four-frequency pure-tone average for air- and bone-conduction thresholds $(0.5,1,2$, and $4 \mathrm{kHz})$. Audiometry was reportedly adapted from American Academy of Otolaryngology Head and Neck Surgery guidelines.[22]

Nasal endoscopic examination was performed for each patient and five nasal anatomic criteria were evaluated for each nasal cavity: congestion and edema of the mucosa, presence of polyps, muco-purulent discharge and crusting. Each criterion was evaluated according to a two-point semiquantitative scale (0:absent; 1 :present). Similarly, otoscopy examination allowed evaluating four otologic anatomic criteria for each ear: sero-mucous otitis, tympanic membrane retraction, chronic otitis media and cholesteatoma. For each patient, a global anatomic score was calculated for the 
nasal cavities and the ears by summing the scores with a maximum rhinologic and otologic anatomic scores of 10 and of 8 , respectively. 


\section{Ciliary investigations}

Biopsies and brushing of ciliated epithelium were obtained from nasal mucosa (inferior turbinate) of the patients and processed for ciliary investigations. All investigations were performed in the absence of acute respiratory tract infections. All the patients and/or their parents were informed of both the exact nature and the goal of the investigations and gave their informed consent as prescribed by the law on bioethics in the European Community (and after approval by the local ethics committee (DC-2008-512, Paris-Necker).

Airway biopsies were immersed in $2.5 \%$ glutaraldehyde and processed as usual for transmission electron (TEM) ultrastructural analysis.[23] Ultrathin sections were examined at a final magnification of 60,000 without knowledge of the clinical data. In each specimen, analysis of at least 50 transverse ciliary sections of different cells were required to study the internal axonemal structure according to a quantitative method.[24] Ciliary ultrastructure results were expressed as a percentage of abnormal cilia among the total number of cilia analyzed. As previously reported, up to $10 \%$ of cilia in control specimens can exhibit ultrastructural defects.[25] For each ciliary study, axonemal abnormalities were expressed as the concerned ultrastructure (i.e. dynein arms, central complex and/or peripheral microtubules).

Airway brushing were suspended in culture medium and processed for ciliary beat pattern evaluation using high-speed videomicrosocopy (HSVM). All the observations were made within three hours at $37^{\circ} \mathrm{C}$ with an inverse microscope (Axiovert 200, Carl Zeiss S.A.S. Le Pecq France) using an oil immersion x100 objective lens on a duration shorter than 20 minutes. Beating ciliated edges were recorded with a highspeed digital camera (PixeLINK A741, Ottawa Canada) at a rate of 355 frames per second. In each patient, we recorded at least 20 distinct areas containing intact undisrupted ciliated epithelial edge greater than $50 \mu \mathrm{m}$ beating in the plane of the camera and devoid of mucus. All isolated ciliated cells were excluded from the study 
as recommended.[26] We initially determined the amount of ciliated cells among epithelial cells and then the percentage of ciliated edges with beating cilia. We then selected a cilium that we followed during an entire cycle of beating in 10 distinct areas at least. For this, the video sequences were played back frame by frame using Streampix software (Norpix Inc., Montreal Canada) on a high resolution monitor. Different beat pattern parameters were determined : cilia length, proportion of cilia with a length $<4 \mu \mathrm{m}$, ciliary beat frequency, beating angle, and running distance per second defined as the distance run by the cilia tip in one second. The cilia length and beating angle were calculated by trigonometric calculations in the triangle made by the anchoring point and the two extreme positions of the cilium extremities during the power stroke. The running distance per second was calculated by combining the cilia length, the ciliary beat frequency and the beating angle.

\section{CEP290 expression in nasal ciliated cells}

We examined the expression of CEP290 transcripts by real-time RT-PCR in adult and foetal human tissues. One microgram of total RNA from sensorineural retina and nasal mucosa extracted from a twenty-week-old fetal human ocular globe and adult control respectively, and, adrenal gland, bone marrow, brain cerebellum, whole brain, fetal brain, fetal liver, heart, kidney, liver, whole lung, placenta, prostate, salivary gland, skeletal muscle, testis, thymus, thyroid gland, trachea, uterus and spinal cord from the Human Total RNA Master panel II (Clontech, Saint Germain en Laye, France), on the other hand, were reverse transcribed with the High capacity cDNA Archive Kit (Applied Biosystems, Foster City, CA) primed with Oligo d(T)16 (Applied Biosystems, Foster City, CA) in accordance with the supplier's recommendations. CEP290 transcript was amplified as $94 \mathrm{bp}$ fragment. Regions of $80 \mathrm{bp}$ and $84 \mathrm{bp}$ within the Homo sapiens beta-2-microglobulin mRNA (B2M, NM_004048.2) and the human glucuronidase beta mRNA (GUSB, NM_000181.3) were used for 
normalization, respectively. Primer sequences were as follows: CEP290 forward 5'TGACTGCTAAGTACAGGGACATCTTG-3' CEP290 reverse 5' AGGAGATGTTTTCACACTCCAGGT-3'B2M forward, 5'- cctggaggctatccagcgtact -3'; B2M reverse, 5'- tcaggaaatttgactttccattctct-3'; GUSB forward, 5'-gcggtcgtgatgtggtctgt -3'; GUSB reverse, 5'- gtgagcgatcaccatcttcaagt -3'.

Samples of cDNA were diluted 1:25 in nuclease-free water (Qiagen, Courtaboeuf, France) and either submitted to qPCR or pooled and further diluted 1:5, 1:25, 1:125, and 1:650 to create standard curves for calculation of relative gene expression levels. Independent or pooled reverse transcribed materials $(5 \mu l)$ were submitted to qPCR in $20 \mu \mathrm{l}$ MESA BLUE qPCR Master Mix Plus for SYBR® Assay (Eurogenetec, Angers, France) containing 300nM of primers, on a Taqman 7900 HT Fast Real-Time PCR System (Applied Biosystems, Foster City, CA) under the following conditions: 1 cycle of $95^{\circ} \mathrm{C}$ for $5 \mathrm{~min}$, followed by 50 cycles of $15 \mathrm{sec}$ at $95^{\circ} \mathrm{C}$ and $1 \mathrm{~min}$ at $65^{\circ} \mathrm{C}$. The specificity and identity of PCR products were verified by generating melting curves using 1 cycle at $95^{\circ} \mathrm{C}$ for $15 \mathrm{sec}, 65^{\circ} \mathrm{C}$ for $15 \mathrm{sec}$ and $95^{\circ} \mathrm{C}$ for $15 \mathrm{sec}$. Data were analyzed using the SDS 2.3 software (Applied Biosystems, Foster City, CA).

For each tissue, three independent reverse transcriptions were performed and each was tested in duplicate. No reverse transcriptase and no template reactions were used as negative controls.

Relative expression values were calculated using the geNorm algorithm.[27]

\section{RESULTS}

\section{Clinical phenotype (Table 1)}

The seven patients displayed a dramatically severe and stationary congenital conerod dystrophy (LCA type I,[18]).

Clinical history revealed many recurrent but moderate inflammatory diseases of the upper and lower airways in six out of the seven patients (Table 1). Clinical 
examination revealed that nasal symptoms were either moderate (four patients) or absent (three patients). A slight impairment of olfaction was present in three patients according to the B-SIT score (Table 1). Nasal endoscopy revealed a slight or moderate inflammation in six patients, but was normal on one patient (Table 1). None of the patients complained of any otological symptom (Table 1). Pure tone audiometry detected a moderate/slight hearing loss on two patients and was normal on the five other patients (Table 1). Eventually, otoscopy was normal on all patients (Table 1).

It was interesting to notice that patient \#5 affected with hypofertility due to oligoasthenospermia, showed normal otorhinolaryngological evaluation, yet slight bilateral sensory hypoacousia.

\section{Ciliary ultrastructure}

Nasal ciliated were sparse in two patients (\#2 and 7) on whom epithelial morphology suggested a partial loss of ciliary differentiation. In these two patients, the low amount of ciliated cells in the biopsy did not allowed to study ciliary ultrastructure. In the other five patients, high levels of abnormal cilia were noted (24-71\%, Table 1$)$. Different ultrastructural defects of the axoneme were evidenced : abnormal number of peripheral and/or central microtubules, either alone or in combination with absence of dynein arms (Table 1; Figure 2).

\section{HSVM quantitative evaluation of ciliary beat pattern}

On all patients, the brushing samples provided enough ciliated cells for ciliary beat pattern evaluation. On all patients, we found a slightly decreased amount of ciliated cells among epithelial cells (Table 1). However, the percentage of ciliated edges with beating cilia was always high (Table 1). On four patients, mean cilia length was reduced and on six patients an increased proportion of short cilia (Table 1) was 
found. The ciliary beat frequency and the beating angle were markedly reduced on two and three patients compared to normal values, respectively (Table 1). Finally, on all patients, the running distance covered by the cilia tip per second was reduced compared to the expected normal value (Table 1). The lower values of running distance were observed on the three patients affected with dynein arm defects (patients 1, 3 and 4). However, there was no obvious relationship between ciliary beat pattern parameters and clinical features. 
Table 1: Ciliated cells characteristics of the LCA patients.

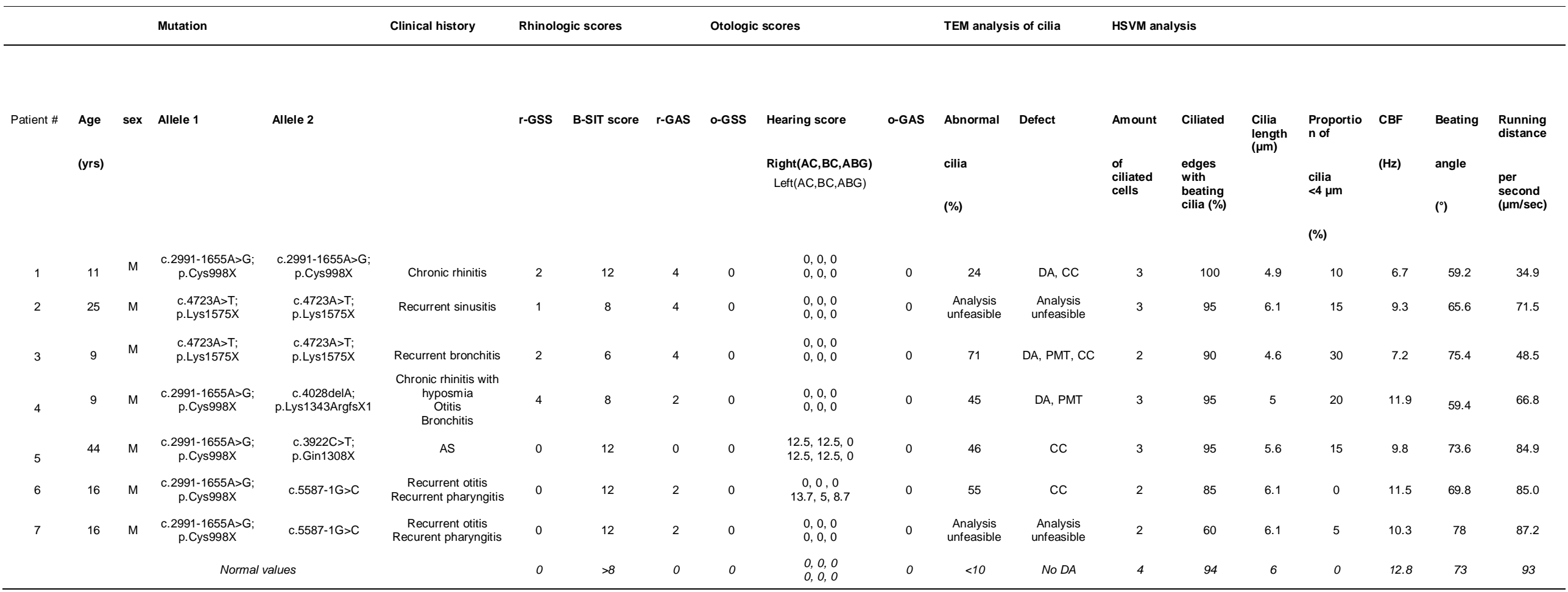

Normal values of the different parameters according to the literature data for B-SIT score,[21] TEM analysis of cilia,[25] amount of ciliated cells and ciliated edges with beating cilia, [28] cilia length,[29] ciliary beat frequency [30] and beating angle.[31] The value of the expected running distance per second was calculated using the following formula: length $\mathrm{x}$ angle $\mathrm{x}$ frequency $\mathrm{x}$ 1second. Abbreviations: TEM: transmission electron microscopy; HSVM:high-speed videomicroscopy; r-GSS: rhinologic global symptom score; B-SIT: brief smell identification test; r-GAS: rhinologic anatomic score; o-GSS: otologic global symptom score; AC: air conduction, BC: bone conduction; ABG: air-bone gap; o-GAS: otologic anatomic score; CBF: ciliary beat frequency; M: male; DA: dynein arms; PMT: peripheral microtubule; CC: central complex; AS: asthenospermia. 


\section{Expression analysis of CEP290 (Figure 2)}

Realtime RT-PCR experiments demonstrated the highest expression levels of CEP290 transcripts in neural retina and nasal epithelium. The gene was also significantly expressed in spinal cord, thyroid gland, testis, heart, lung, bone marrow, cerebellum and uterus. Weaker expression was noted in whole brain, both the foetal brain and the kidney. Finally trachea, thymus, muscle, salivary gland, liver and placenta expressed very low levels of transcripts (Figure 2).

\section{DISCUSSION}

Leber congenital amaurosis is characterized by a large genetic and pathophysiologic heterogeneity. However, until the recent identification of CEP290, the specific or preferential pattern of expression of LCA genes was consistent with the LCA retinalrestricted phenotype. Intriguingly, while the implication of CEP290 in syndromic LCA was consistent with its expression in primary cilia of various cell types, its involvement in non-syndromic LCA remains unexplained. Interestingly, here, we report abnormalities of motile respiratory cilia with frequent respiratory history that were consistently found in LCA patients with homozygote or compound heterozygote for loss-of-function or the common intronic CEP290 mutations.

Respiratory symptoms were reported by all but one LCA patients. Patients with otorhinolaryngologic history complained of rhinorrhea and nasal obstruction with inflammatory nasal mucosa, highly suggestive of chronic rhinitis. In addition, two of them (\#3 and 4) also complained of slight bronchial symptoms. Motile cilia were studied at nasal level because ciliated cells are easily collected after local anesthesia, through a non-invasive procedure routinely used to investigate chronic rhinitis.[32] Moreover, we previously demonstrated that the ciliary defects present in nasal mucosa are identical to those seen in bronchi.[33] In the five patients on whom 
TEM analysis was possible, we detected a high proportion of cilia with axonemal structural defects affecting, alone or in combination, dynein arms, peripheral and/or central microtubules. These data suggest the constitutional nature of the ciliary abnormalities rather than a consequence of acquired ciliary defects resulting from recurrent infections which usually only affect a few cilia and do not involve dynein arms.[34]

Along with axonemal defects, HSVM evidenced a rarefaction of ciliated cells with frequent short cilia. As a result, the distance covered by the ciliary tip per second was consistently shortened in all patients, especially in patients with dynein arm defects. This latter observation is consistent with the pivotal role of dynein arms in the transduction of mechanical forces necessary for ciliary motion via a number of complex cell events including phosphorylation/dephosphorylation of key proteins.[1] The ciliary phenotype documented in the patients (i.e. rarefaction and short cilia, partial ciliary defects with abnormalities of several axonemal components) is consistent with alterations of ciliary construction and/or maintenance. The high expression level of CEP290 in nasal mucosa along with the recent report of its pivotal role in primary cilia assembly [35] suggest that the respiratory cilia defects in patients may be related to the CEP290 mutations. In this context, the coexistence of normal and abnormal motile cilia in nasal samples of patients may reflect differences in assembly pathways of primary versus motile cilia.

It would be tempting to correlate the mild symptoms in patients to the moderate though significant decrease in the amount of ciliated cells and the increase in abnormal structural defects of cilia in the nasal mucosa.

Interestingly, despite nasal congestion or edema, patients of our series, including that homozygote for the CEP290 c. $2991+1655 A>$ G mutation, had quite a normal sense of smell. Three patients (\#2, 3 and 4) at best failed to identify some flavors. This finding differs from the severe olfactory impairment reported in patients of a large inbred 
family homozygous for the "hypomorphic" CEP290 mutation and in rd16 mice carrying an in-frame CEP290 deletion mutation.[21]

Finally, none of the seven patients complained about hearing or eardrum abnormalities. Audiogram recordings however indicated slight/mild hearing loss in $2 / 7$ patients (\#5 and \#6) which may result from otitis in infancy.

The variation among patients of extraocular symptoms as well as in the proportion of abnormal cilia and/or the axonemal ultrastructural defects in upper-airways did not correlated to their genotype:

i) similar clinical findings were noted in patients homozygote for loss-offunction or the common intronic mutations,

ii) patients with same genotypes presented with variable clinical symptoms and/or variable proportions of abnormal cilia

iii) the three patients with dynein arm defects and ciliary beating abnormalities exhibited different genotypes.

These findings may be compared to those reported previously in patients harboring RPGR mutations.[16] However, in some families with $R P G R$ mutations, a broader phenotype was reported in which RP and sensorineural hearing loss also included ear and respiratory tract infections with inconstant anosmia or congenital pulmonary abnormalities (c.845-846delTG [17]; p.G173R,[14]). Moreover, RPGR mutations were also reported in patients with a complex $\mathrm{X}$ linked phenotype combining RP and primary ciliary dyskinesia.[16]

In conclusion, the data presented here provide a first report of respiratory cilia abnormalities in LCA patients harbouring CEP290 mutations, supporting the classification of some forms of the disease in the increasing family of ciliopathies.

Owing to the high frequency of CEP290 mutations in LCA (ca. 17\%), this symptomatic yet inconstant association might be frequent and largely overlooked. The description of otorhinolaryngologic history in patients could represent additional 
clinical criteria to direct CEP290 genotyping of patients affected with the genetically heterogeneous cone-rod dystrophy subtype of LCA. Further studies will however be necessary to compare the frequency of otorhinolaryngologic history in patients with CEP290 and other disease genes. Finally, in the future, one can wonder whether the defects identified in respiratory cilia could be used as useful markers in the development of genetic therapies for LCA.

\section{AKNOWLEDGEMENTS}

The authors are grateful to the patients and their families who participated in this study. We would also like to thank Dr V. Chatelin for the audiometric evaluation of the patients.

This work was supported by grants and sponsors from the Legs Poix from the Chancellerie des Universites, the Assistance Publique-Hopitaux de Paris (PHRC AOM06053, P060245), the Agence Nationale pour la Recherche (ANR-05-MRAR022-01), EVI-Genoret (LSHG-CT-2005-512036), the Foundation Fighting Blindness (BR-GE-0406-0335) and the Association Retina France.

\section{REFERENCES}

1. Haimo LT, Rosenbaum JL. Cilia, flagella, and microtubules. J Cell Biol $1981 ; 1991: 125 s-130 s$

2. Rosenbaum JL, Witman GB. Intraflagellar transport. Nat Rev Mol Cell Biol 2002;3:813-825.

3. Marshall WF. The cell biological basis of ciliary disease. I Cell Biol 2008;180:17-21.

4. Adams NA, Awadein A, Toma HS. The retinal ciliopathies. Ophthalmic Genet 2007;28:113-125. 
5. Roepman R, Wolfrum U. Protein networks and complexes in photoreceptor cilia. Subcell Biochem 2007;43:209-235.

6. Xi Q, Pauer GJ, Ball SL, et al. Interaction between the photoreceptor-specific tubby-like protein 1 and the neuronal-specific GTPase dynamin-1. Invest Ophthalmol Vis Sci 2007;48:2837-2844.

7. den Hollander Al, Koenekoop RK, Yzer S, et al. Mutations in the CEP290 (NPHP6) gene are a frequent cause of Leber congenital amaurosis. Am J Hum Genet 2006;79:556-561.

8. Den Hollander Al, Koenekoop RK, Mohamed MD, et al. Mutations in LCA5, encoding the ciliary protein lebercilin, cause Leber congenital amaurosis. Nat Genet 2007;39:889-895.

9. Gerber S, Hanein S, Perrault I, et al. Mutations in LCA5 are an uncommon cause of Leber congenital amaurosis (LCA) type II. Hum Mutat 2007;28:1245.

10. Baala L, Audollent S, Martinovic J, et al. Pleiotropic effects of CEP290 (NPHP6) mutations extend to Meckel syndrome. Am J Hum Genet 2007;81:170-179.

11. Sayer JA, Otto EA, O'Toole JF, et al. The centrosomal protein nephrocystin-6 is mutated in Joubert syndrome and activates transcription factor ATF4. Nat Genet 2006;38:674-681.

12. Valente EM, Silhavy JL, Brancati $F$, et al. Mutations in CEP290, which encodes a centrosomal protein, cause pleiotropic forms of Joubert syndrome. Nat Genet 2006;38:623-625.

13. Perrault I, Delphin N, Hanein S, et al. Spectrum of NPHP6/CEP290 mutations in Leber congenital amaurosis and delineation of the associated phenotype. Hum Mutat 2007;28:416.

14. Iannaccone A, Breuer DK, Wang XF, et al. Clinical and immunohistochemical evidence for an $X$ linked retinitis pigmentosa syndrome with recurrent 
infections and hearing loss in association with an RPGR mutation 2003;40:509-515.

15. Koenekoop RK, Loyer M, Hand CK, et al. Novel RPGR mutations with distinct retinitis pigmentosa phenotypes in French-Canadian families. $A m \mathrm{~J}$ Ophthalmol 2003;136:678-687.

16. Moore A, Escudier E, Roger G, et al. RPGR is mutated in patients with a complex $\mathrm{X}$ linked phenotype combining primary ciliary dyskinesia and retinitis pigmentosa. J Med Genet 2006;43:326-333.

17. Zito I, Downes SM, Patel RJ, et al. RPGR mutation associated with retinitis pigmentosa, impaired hearing, and sinorespiratory infections. J Med Genet 2003;40:609-615.

18. Perrault I, Rozet JM, Gerber S, et al. Leber congenital amaurosis. Mol Genet Metab 1999;68:200-208.

19. Coste A, Yona L, Blumen M, et al. Radiofrequency is a safe and effective treatment of turbinate hypertrophy. Laryngoscope 2001;111:894-899.

20. Doty RL, Marcus A, Lee WW. Development of the 12-item Cross-Cultural Smell Identification Test (CC-SIT). Laryngoscope 1996;106:353-356.

21. McEwen DP, Koenekoop RK, Khanna H, et al. Hypomorphic CEP290/NPHP6 mutations result in anosmia caused by the selective loss of $\mathrm{G}$ proteins in cilia of olfactory sensory neurons. Proc Natl Acad Sci U S A 2007;104:1591715922.

22. Committee on Hearing and Equilibrium guidelines for the evaluation of results of treatment of conductive hearing loss. AmericanAcademy of OtolaryngologyHead and Neck Surgery Ffoundation, Inc. Otolaryngol Head Neck Surg $1995 ; 113: 186-187$.

23. Papon JF, Coste A, Roudot-Thoraval F, et al. A 20-year experience of electron microscopy in the diagnosis of primary ciliary dyskinesia. Eur Respir J 2009. 
24. Escalier D, Jouannet P, David G. Abnormalities of the ciliary axonemal complex in children: an ultrastructural and cinetic study in a series of 34 cases. Biol Cell 1982;44:271-282.

25. de longh RU, Rutland J. Ciliary defects in healthy subjects, bronchiectasis, and primary ciliary dyskinesia. Am J Respir Crit Care Med 1995;151:15591567.

26. Thomas B, Rutman A, O'Callaghan C. Disrupted ciliated epithelium shiws slower ciliary beat frequency and increased dyskinesia. Eur Respir $J$ 2009;34:401-404.

27. Vandesompele J, De Preter K, Pattyn F, et al. Accurate normalization of realtime quantitative RT-PCR data by geometric averaging of multiple internal control genes. Genome Biol 2002;3:RESEARCH0034.

28. Rossman C, Lee R, Forrest $\mathrm{J}$, et al. Nasal ciliary ultrastructure and function in patients with primary ciliary dyskinesia compared with that in normal subjects and in subjects with various respiratory diseases. Am Rev Respir Dis 1984;129:161-167.

29. Sleigh MA, Blake JR, Liron N. The propulsion of mucus by cilia. Am Rev Respir Dis 1988;137:726-741.

30. Chilvers MA, Rutman A, O'Callaghan C. Functional analysis of cilia and ciliated epithelial ultrastructure in healthy children and young adults. Thorax 2003;58:333-338.

31. Rautiainen M, Matsune S, Shima S, et al. Ciliary beat of cultured human respiratory cells studied with differential interference microscope and high speed video system. Acta Otolaryngol 1992;112:845-851.

32. Chapelin C, Coste A, Gilain L, et al. Modified epithelial cell distribution in chronic airways inflammation. Eur Respir J 1996;9:2474-2478. 
33. Verra F, Fleury-Feith J, Boucherat M, et al. Do nasal ciliary changes reflect bronchial changes? An ultrastructural study. Am Rev Respir Dis 1993;147:908-913.

34. Leigh MW, Pittman JE, Carson JL, et al. Clinical and genetic aspects of primary ciliary dyskinesia/Kartagener syndrome. Genet Med 2009;11:473-487.

35. Tsang WY, Bossard C, Khanna $\mathrm{H}$, et al. CP110 suppresses primary cilia formation through its interaction with CEP290, a protein deficient in human ciliary disease. Dev Cell 2008;15:187-197.

\section{LEGENDS TO FIGURES}

Figure 1. Examples of ultrastructural defects of cilia observed in patients with LCA (magnification $\times 60,000$; bars $=0.1 \mu \mathrm{m}$ ).

A: control normal cilia;

B: partial absence of inner dynein arms associated with abnormal central complex (patient1)

C: partial absence of both dynein arms associated with abnormal central sheath (patient 3)

D: absence of both dynein arms associated with supernumerary microtubules (patient 4)

$\mathrm{E}$ and $\mathrm{F}$ : absence of central complex (patients 5 and 6 , respectively)

Figure 2. Relative expression level of the CEP290 transcript in different human tissues. Housekeeping genes: TBP and RPLO. 


\section{Licence for Publication statement}

"I Jean-Michel ROZET, the Corresponding Author has the right to grant on behalf of all authors and does grant on behalf of all authors, an exclusive licence (or non exclusive for government employees) on a worldwide basis to the BMJ Publishing Group Ltd to permit this article (if accepted) to be published in JMG and any other BMJPGL products and sublicences such use and exploit all subsidiary rights, as set out in our licence (http://group.bmj.com/products/journals/instructions-forauthors/licence-forms)." 
A

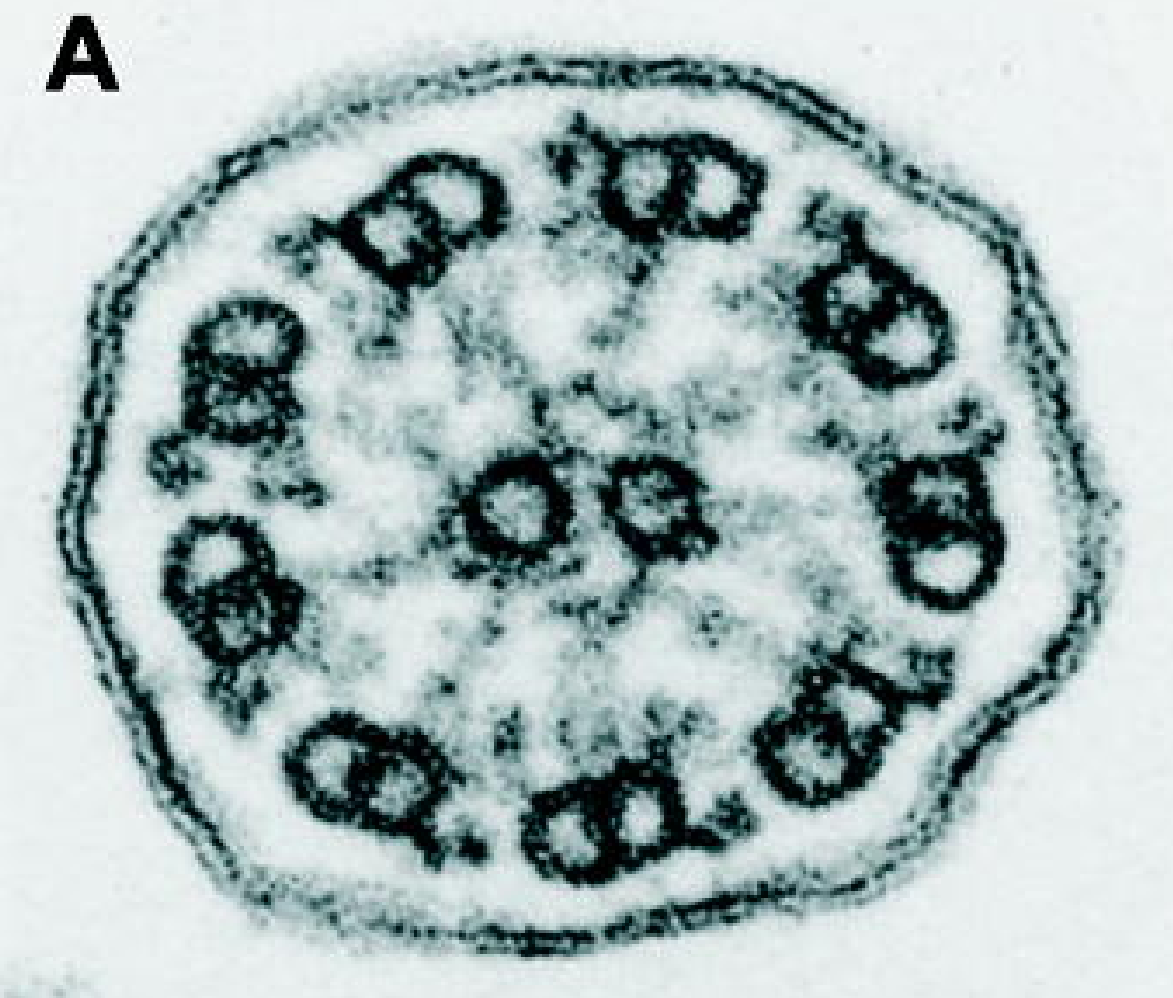

B

D

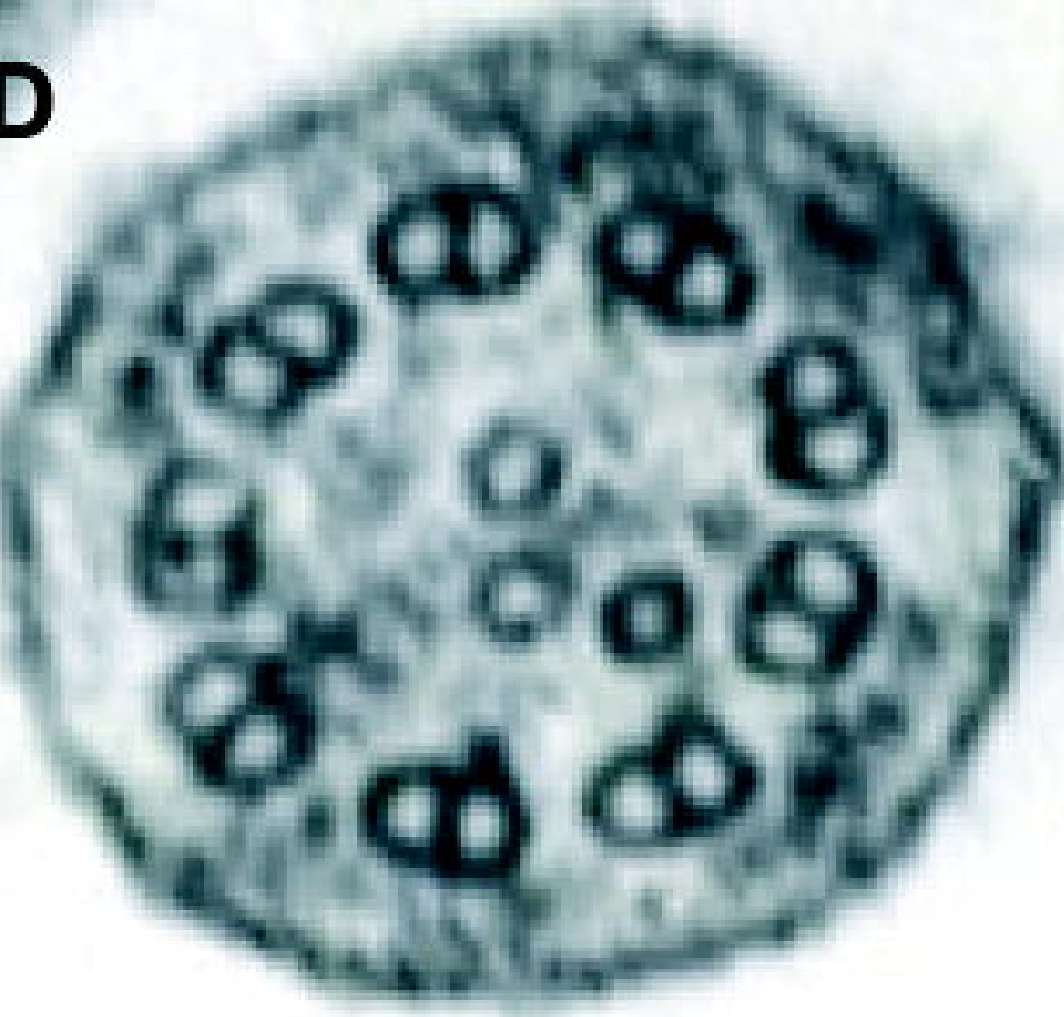

C

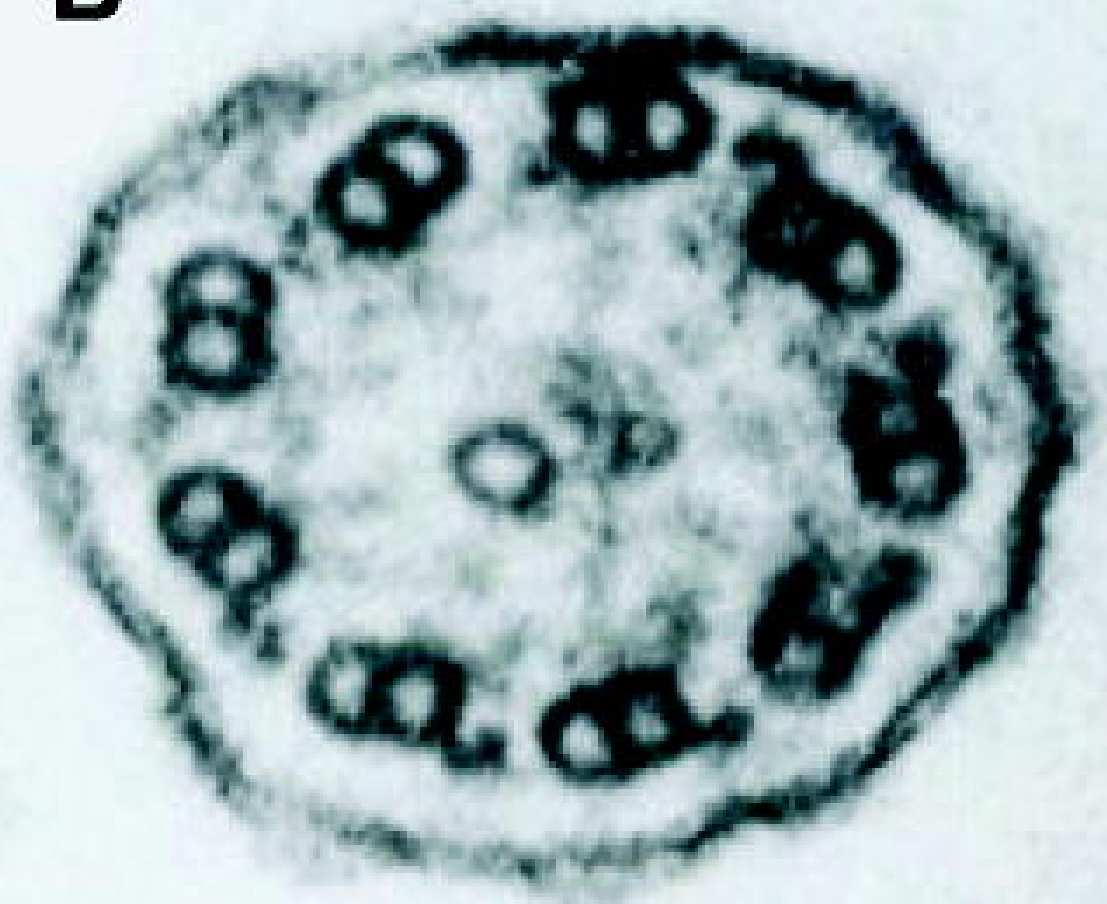

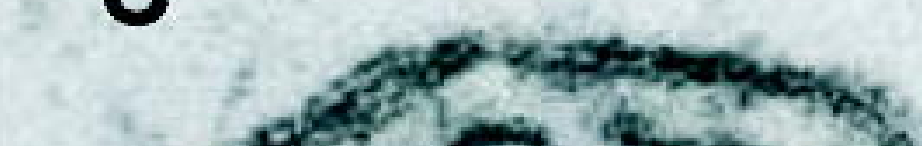

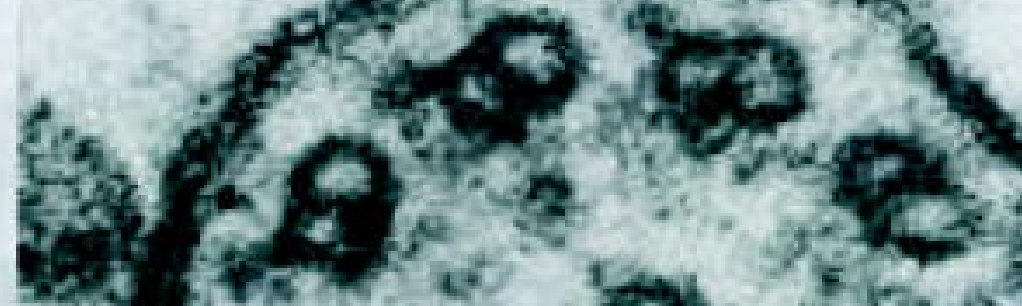

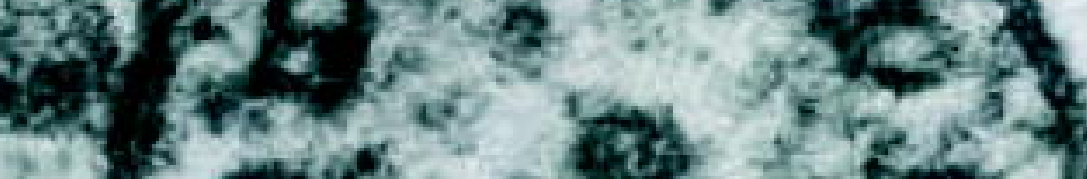

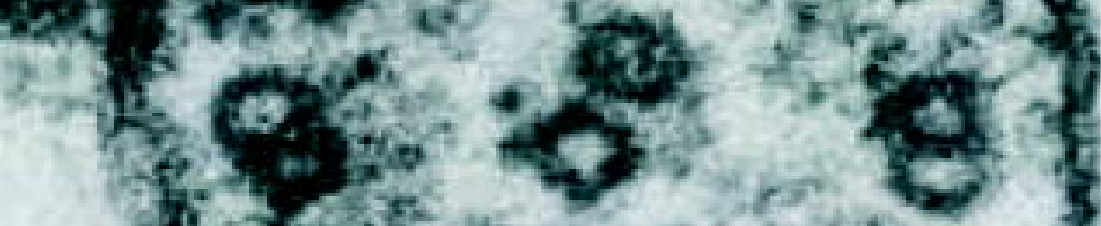

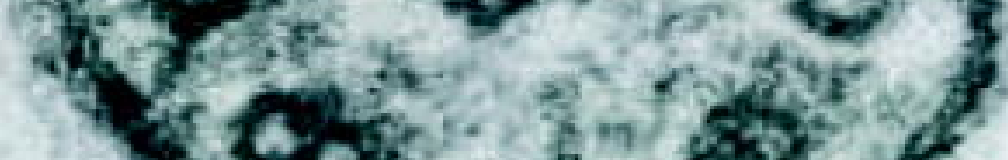
(4) 68 in: - 4hor cos: adont?

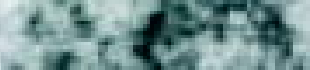
203246

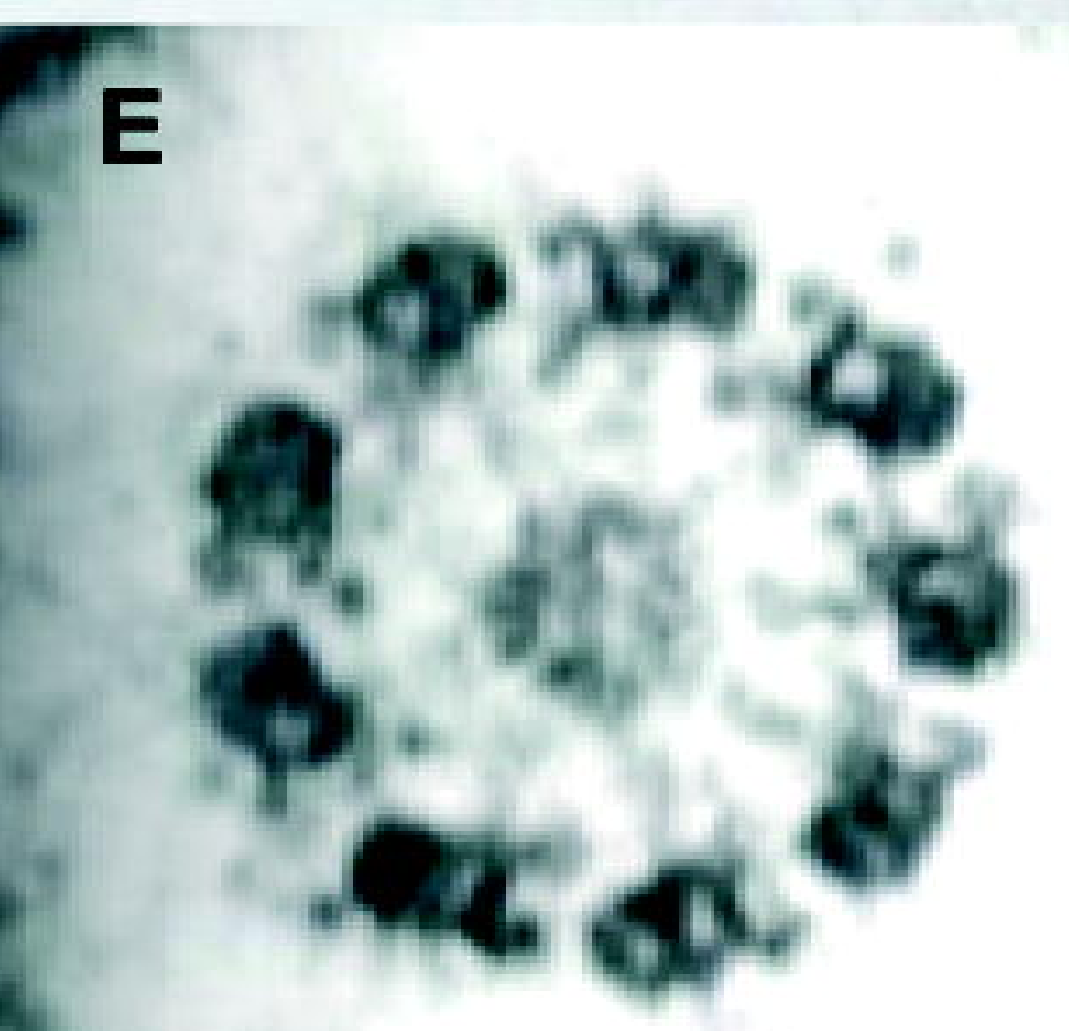<smiles>C=[Te]</smiles>

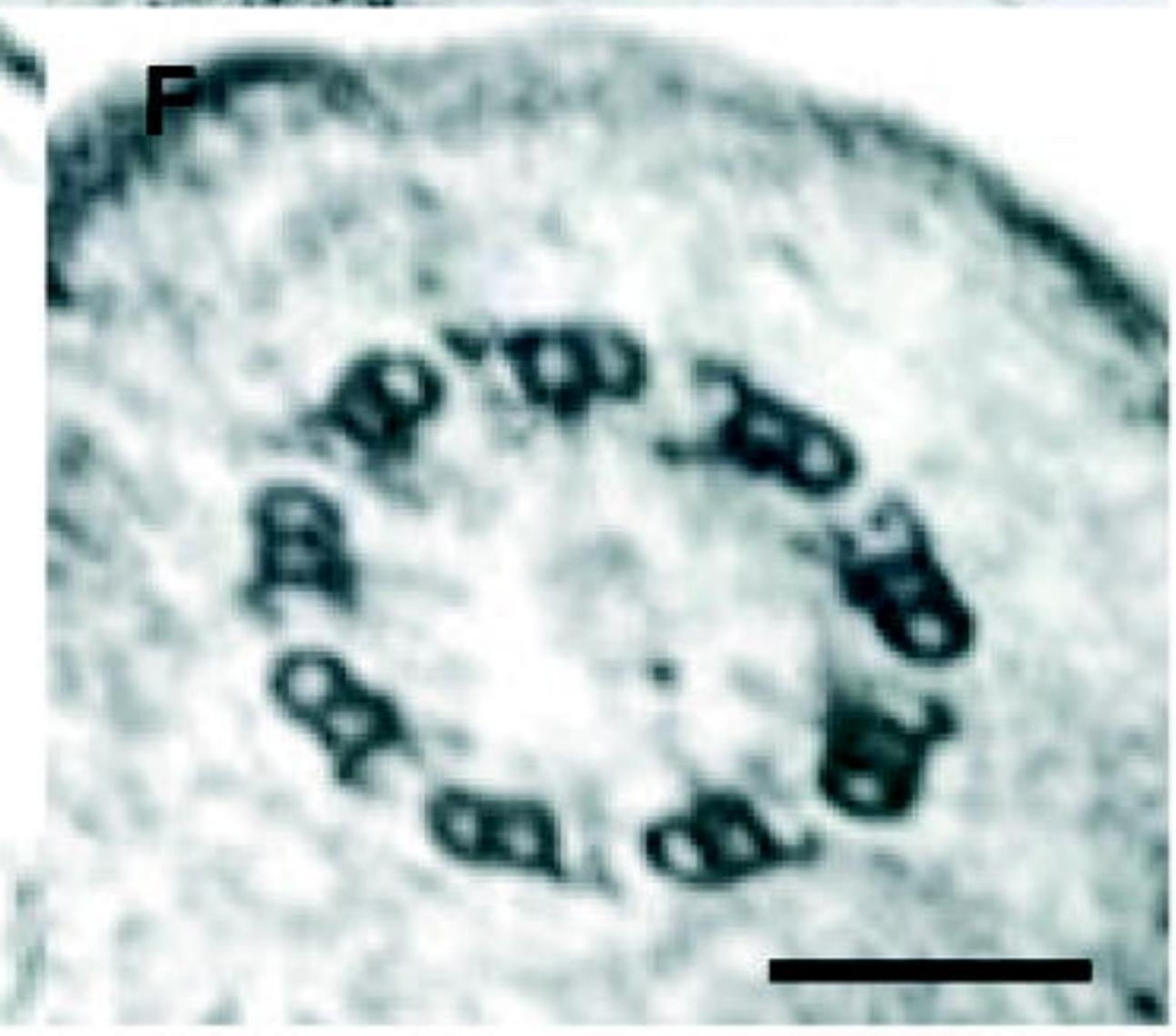




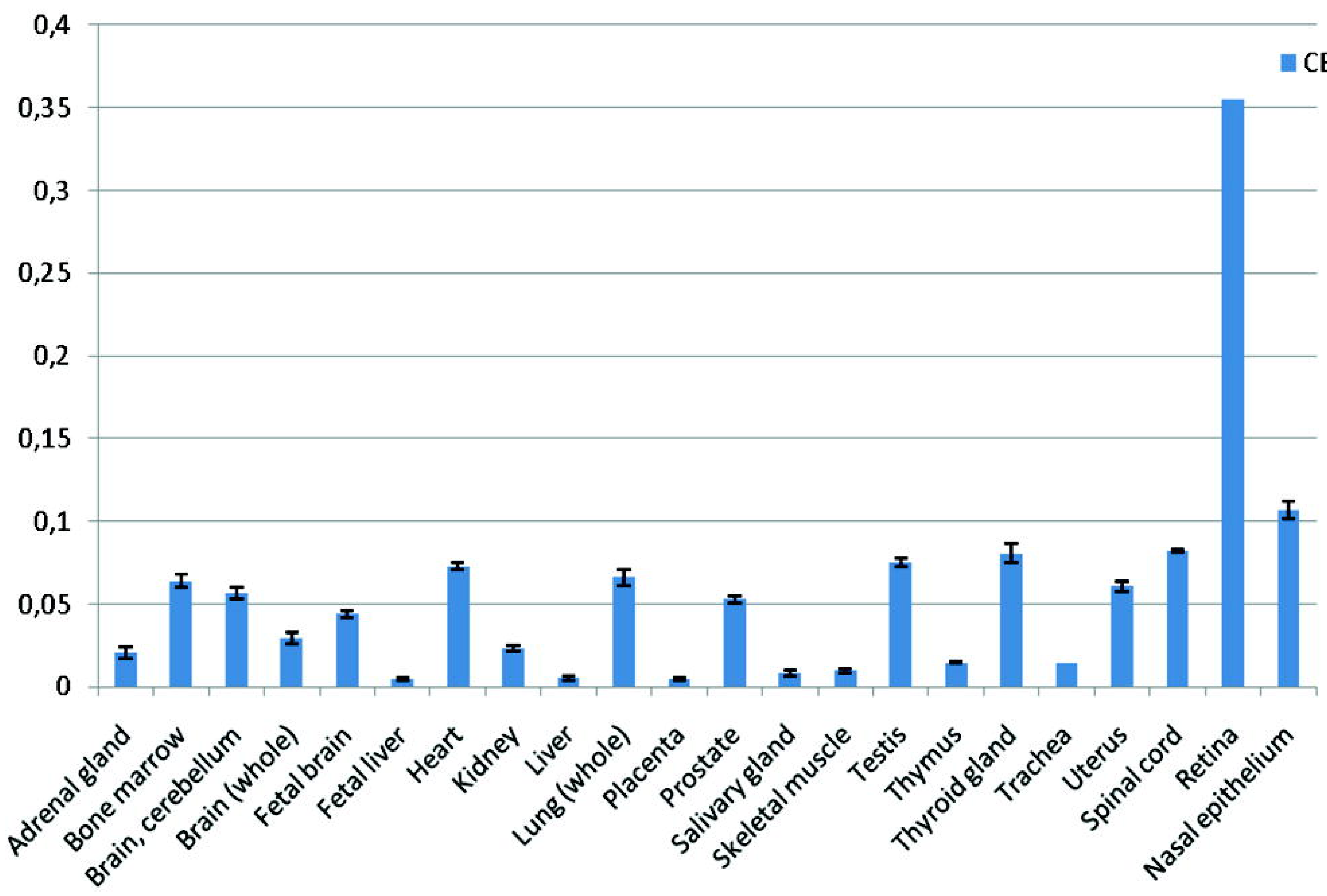

